\title{
The Quasicrystal Model as a Framework for Order to Disorder Transitions in 2D Systems ${ }^{+}$
}

\author{
Nadezhda L. Cherkas ${ }^{1}$ and Sergey L. Cherkas ${ }^{2, *}$ \\ 1 Military Academy of the Republic of Belarus, 220057 Minsk, Belarus; n_cherkas@mail.ru \\ 2 Institute for Nuclear Problems, Belarus State University, 220030 Minsk, Belarus \\ * Correspondence: cherkas@inp.bsu.by; Tel.: +375-17-226-47-39 \\ + Presented at the 1st International Electronic Conference on Crystals, 21-31 May 2018. Available online: \\ https://sciforum.net/conference/IECC_2018.
}

Published: 21 May 2018

\begin{abstract}
Order to disorder transitions are important for 2D objects such as oxide films with a cellular porous structure, honeycomb, graphene, and Bénard cells in liquid and artificial systems consisting of colloid particles on a plane. For instance, solid films of the porous alumina represent an almost regular quasicrystal structure (perfect aperiodic quasicrystals discovered in 1991 is not implied here). We show that, in this case, the radial distribution function is well described by the quasicrystal model, i.e., the smeared hexagonal lattice of the two-dimensional ideal crystal by inserting a certain amount of defects into the lattice. Another example is a system of hard disks in a plane, which illustrates the order to disorder transitions. It is shown that the coincidence with the distribution function, obtained by the solution of the Percus-Yevick equation, is achieved by the smoothing of the square lattice and injecting the defects of the vacancy type into it. However, a better approximation is reached when the lattice is a result of a mixture of the smoothened square and hexagonal lattices. Impurity of the hexagonal lattice is considerable at short distances. Dependences of the lattices constants, smoothing widths, and impurity on the filling parameter are found. Transition to the order occurs upon an increasing of the hexagonal lattice contribution and decreasing of smearing.
\end{abstract}

Keywords: order to disorder transitions; 2D objects; radial distribution function; quasicrystal model; wavelet; porous aluminium oxide; hard disks in a plane

\section{Introduction}

It is well known that for particles on a plane interacting via a certain potential, the existence of crystal, i.e., a system with periodicity, is impossible at a nonzero temperature [1-4]. However, there exist a number of 2D objects in nature which exhibit crystalline-like ordering. Usually, these objects have a non-perfect crystalline structure and a question arises about the transition from order to disorder. It is noteworthy that the order-disorder transition was experimentally studied from the general point of view in [5], where the destruction of a graphene layer by the electron beam was analyzed. A continuous transition from the crystalline state to the glassy state occurred as a result of irradiation. At the initial stage of irradiation, individual defects appeared in the lattice. Then, the glassy (disordered) regions surrounding crystallites (perfectly oriented regions) emerged. In the disordered regions, the destruction of the order initially manifested itself as pentagonal and heptagonal cells instead of the hexagonal ones. The area occupied by crystallites decreased with an increase in the irradiation dose.

As it was believed in [5], an experiment confirms the validity of both of the two competing theories of the order-disorder transition. They involve, on the one hand, the theory of crystallites [6] 
(according to which an amorphous substance incorporates crystal-like agglomerates linked by disordered regions) and, on the other hand, the theory of random networks (see review [7]), which assumes that the order is destroyed throughout the entire volume of a substance, so that the crystal lattice transforms into random chains of atoms due to the distortion and breakage of some bonds. Thus, it follows from the experiment [5] that single defects are formed at the initial stages. Then, the theory of crystallites proves to be valid; further, the theory of random networks starts to work. In this study, we propose to take into consideration two mechanisms in order to simulate the radial distribution function of 2D systems. The first implies the formation of vacancy-type defects (i.e., the absence of atoms in some lattice sites), when the crystal lattice structure is retained. The second mechanism corresponds to the destruction of the crystal lattice and is implemented via peak broadening in the structure factor and limitation of the peak height at large wave numbers. The method of crystal lattice smearing proposed in [8] was used to construct an empirical radial distribution function. This method was applied to describe the structure of some simple liquids [9-15]. A similar method of smearing of coordination circles was used to describe artificial 2D crystals $[16,17]$. Here, we consider a slightly different method consisting of the random shifting of the crystal nodes.

\section{Radial Distribution Function for Porous Aluminum Oxide Layer}

Oxide films with a cellular porous structure are formed upon the electrochemical oxidation (anodization) of aluminum and many aluminum alloys in solutions of various acids. The film thickness may be as large as several hundreds of micrometers, while the pore radius size is several tens of nanometers. Such films have been studied for more than 50 years. They are widely applied as anticorrosion, wear-resistant, electro-insulating, and decorative coatings in microelectronic and optical devices, membranes, and sensors. Under a combination of certain conditions, including acid concentration and type, a film can be grown as an ordered array of cylindrical pores similar to a 2D periodic lattice [18-20]. Various theories describing the ordered structure of pores in aluminum oxide fabricated by anodization have been proposed [21,22]. However, presently, there is no complete understanding of the regularities of ordered structure formation; hence, the radial distribution function of pores cannot be calculated theoretically. At the same time, the knowledge of the radial distribution function is necessary for optical calculations. For example, the effective refractive index of a medium consisting of porous aluminum oxide strongly depends on the radial distribution function [23]. The imaginary part of the effective refractive index is particularly sensitive to the form of the radial distribution function. As for the experimental determination of the radial distribution function using an electron microscope, its accuracy is limited by the finite sizes of a sample [24]. Thus, one needs semi-empirical models to describe the radial distribution function of pores in porous aluminum oxide. It is reasonable to suggest that the ordered structure of porous aluminum oxide is something intermediate between the 2D crystal and amorphous substance. Therefore, it is meaningful to study porous aluminum oxide from the viewpoint of general principles of the order-disorder transition $[25,26]$.

Let us first analyze a real sample of porous aluminum oxide in order to understand the form of the radial distribution function that should be sought after. In practice, there is generally a photograph of the sample, which can be analyzed by statistical methods [24]. In this study, we used the electron microscopy image obtained in [27] and shown in Figure 1a. There are computer programs for determining the centers of cylindrical holes [24], which analyze the contrast of pixels. However, we plotted the pore centers manually. The average distance between pores in the sample was in the order of $100 \mathrm{~nm}$ [27]. To avoid edge effects and, at the same time, to have an opportunity to calculate the radial correlation function at somewhat larger distances, we increased the sample area by parallel translations along the coordinate axes and diagonals (Figure 1b). Although this increase in the area gave rise to a certain error, it allowed increasing the distance at which the correlation function can be calculated. Then, using a computer program, we counted the number of pores between the circles of radii $\rho$ and $\rho+d \rho$ (including the pores of the extended sample) in the neighborhood of each selected pore of the initial sample. Such a calculation was performed for 
each pore of the initial sample, and then the average value was found. A binary distribution function of the pore density distribution was calculated by the formula

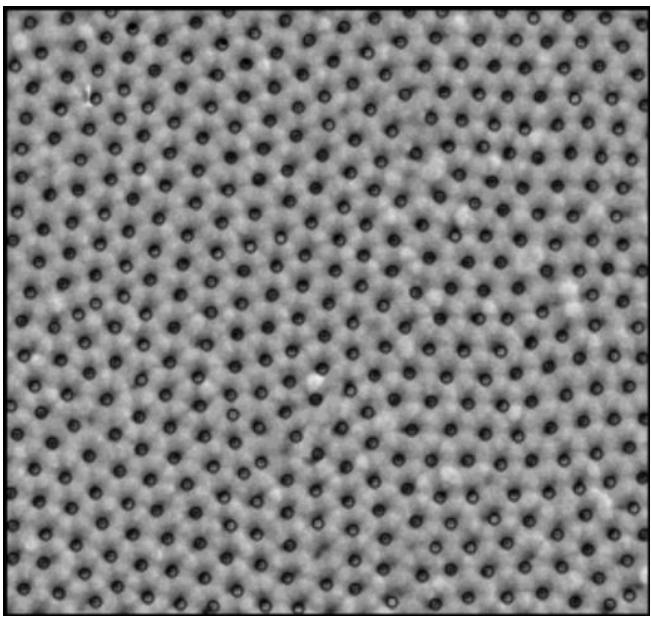

(a)

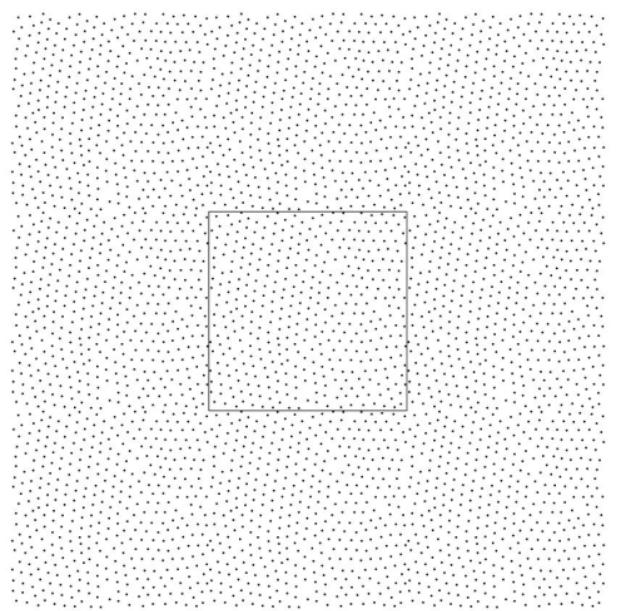

(b)

Figure 1. (a) Photograph of the sample of the porous aluminum oxide layer from Ref. [8]. Circles around pore centers are plotted; (b) Array of pore centers obtained by a parallel translation of the pore centers in the initial array along the coordinate axes and the diagonals of the sample. The region containing pore centers in the starting sample is selected by a rectangle.

$$
g(\rho)=\frac{\Delta N}{\pi\left((\rho+\Delta \rho)^{2}-\rho^{2}\right)} \frac{N}{N+1},
$$

where $\Delta N$ is the number of pores in the region between the circles of radii $\rho$ and $\rho+d \rho$, whereas $N$ is the number of pores in the sample. The correction factor $N /(N+1)$ in (1) takes into account the fact that the selected pore cannot be situated within its environment. The radial distribution function $W(\rho)$ related to the function $g(\rho)$ by the expression $W(\rho)=g(\rho) / n_{0}$ is usually used, where $n_{0}$ is the average surface density of pores in the sample. Thus, the calculation of the radial distribution function is similar to the calculation in the Monte Carlo method, except for the fact that the sample in the Monte Carlo method is generated, while the method at hand deals with an experimental sample. As a result, the radial distribution function was obtained. However, the statistical uncertainty and the error due to edge effects restrict the direct use of this function for optical calculations, since they give rise to errors in the calculation of integrals. At the same time, this accuracy is quite sufficient to construct an empirical distribution function by comparison with the experimental one. A further objective of our study was to derive a smooth empirical distribution function, which could be used in optical calculations. A starting point of our approach is the binary distribution function of particles for an ideal 2D crystal with a certain hexagonal lattice, shown in Figure 2a. The crystal structure is described, e.g., in $[16,25,26]$. The two-particle distribution function $\mathrm{g}(\mathrm{r})$ is a sum of the Dirac delta-function terms:

$$
\tilde{g}(\boldsymbol{\rho})=\sum_{i} \delta^{(2)}\left(\boldsymbol{\rho}-\boldsymbol{\rho}_{i}\right),
$$

where $\delta^{(2)}(\boldsymbol{\rho})$ represents a two-dimensional delta-function by Dirac, and $\boldsymbol{\rho}_{i}$ represents the position vectors of particles in an ideal lattice. The particles are situated in the nodes of the crystalline lattice. Summation in Formula (2) is performed over all the particles, except one, being in an origin of coordinates. As we have one particle per a cell, an average density of the particles in an ideal crystal is easily calculated: $\tilde{n}_{0}=\frac{1}{S}=\left(\frac{m a^{2}}{4} \tan \frac{\pi}{m}\right)^{-1}$, where $S$ is the area of a crystal cell; $a$ is 
a lattice constant, i.e., diameter of a circle inscribed into the cell, as it is shown in Figure 2; and $m$ is the number of edges of a cell.

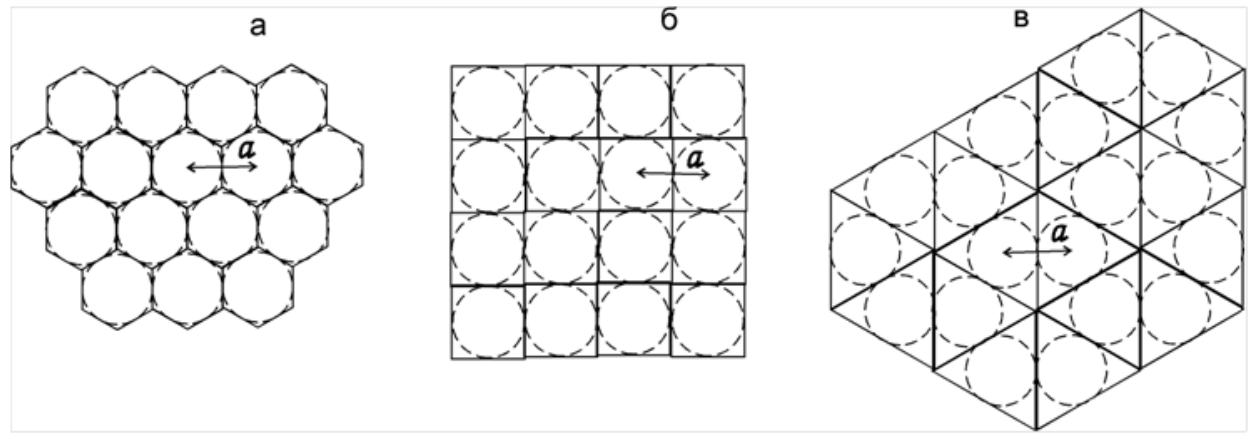

(a) (b) (c)

Figure 2. The two-dimensional lattices: hexagonal (a), square (b), triangular (c).

The correlation function is denoted as $\tilde{K}(\boldsymbol{\rho})=\tilde{g}(\boldsymbol{\rho})-\tilde{n}_{0}$. To proceed from order (an ideal crystal) to disorder, we suggest the formation of the defects of the vacancy type at the first stage. This means that there exists a probability of the lack of a particle in a node. As a result, the distribution function takes the form

$$
\tilde{\tilde{g}}(\boldsymbol{\rho})=\left\{\begin{array}{cc}
n_{0}+\tilde{\tilde{K}}(\boldsymbol{\rho})=n_{0}+\left(\sum_{i} \delta^{(2)}\left(\boldsymbol{\rho}-\mathbf{\rho}_{i}\right)-\tilde{n}_{0}\right) e^{-\lambda\left(\rho-D_{\min }\right)}, & \rho>D_{\min }, \\
0 \quad, & \rho<D_{\min },
\end{array}\right.
$$

where $\tilde{\tilde{g}}(\boldsymbol{\rho})$ denotes the distribution function at this first stage, $n_{0}$ is the surface density of the particles in a sample, and $D_{\min }$ is the distance at which it is known that the distribution function is apparently zero. Formula (3) suggests that the correlation function $\tilde{K}(\boldsymbol{\rho})$ of an ideal crystal is exponentially restricted at a large $\rho$ value. Besides, it is taken into account that $\tilde{\tilde{g}}(\boldsymbol{\rho})$ should have the correct asymptotic at infinity, where it is equal to $n_{0}$, and should be equal to zero at $\rho<D_{\min }$. At the second stage, the spreading of a lattice by means of some integral transformation is carried out:

$$
g(\boldsymbol{\rho})=\int_{\rho^{\prime}>D_{\min }} G\left(\boldsymbol{\rho}, \boldsymbol{\rho}^{\prime}\right) \tilde{\tilde{g}}\left(\boldsymbol{\rho}^{\prime}\right) \mathbf{d}^{2} \mathbf{\rho}^{\prime}, \quad \rho>D_{\min },
$$

where we supposedly have

$$
G\left(\boldsymbol{\rho}, \boldsymbol{\rho}^{\prime}\right)=\Delta(\rho) \exp \left(-\frac{\left(\boldsymbol{\rho}^{\prime}-\boldsymbol{\rho}\right)^{2}}{2 \sigma^{2}(\rho)}\right)
$$

The integral transformation given by Equations (4) and (5) maps a set of the integrable functions defined in a two-dimensional region $\rho>D_{\min }$ into itself. At $\rho<D_{\min }, g(\boldsymbol{\rho})=0$, as in (3). Normalising factor $\Delta(\rho)$ is equal to

$$
\Delta(\rho)=\left(\int_{\rho^{\prime}>D_{\min }} \exp \left(-\frac{\left(\boldsymbol{\rho}^{\prime}-\boldsymbol{\rho}\right)^{2}}{2 \sigma^{2}(\rho)}\right) d^{2} \boldsymbol{\rho}^{\prime}\right)^{-1}=\left(2 \pi \int_{D_{\min }}^{\infty} \exp \left(-\frac{\left(\rho^{\prime 2}+\rho^{2}\right)}{2 \sigma^{2}(\rho)}\right) I_{0}\left(\frac{\rho^{\prime} \rho}{\sigma^{2}(\rho)}\right) \rho^{\prime} d \rho^{\prime}\right)^{-1},
$$

where $I_{0}(z)$ is a zero order modified Bessel function of the first kind. The above expression for $\Delta(\rho)$ implies that the action of the transformation (4) action to the function equal to some constant does not change it. As a result, one comes to 


$$
\begin{aligned}
& g(\boldsymbol{\rho})=n_{0}+\Delta(\rho)\left(\sum_{i} \exp \left(-\lambda\left(\rho_{i}-D_{\min }\right)-\frac{\left(\boldsymbol{\rho}_{i}-\boldsymbol{\rho}\right)^{2}}{2 \sigma^{2}(\rho)}\right)-\right. \\
& \left.-2 \pi \tilde{n}_{0} \int_{D_{\min }}^{\infty} \exp \left(-\lambda\left(\rho^{\prime}-D_{\min }\right)-\frac{\left(\rho^{\prime 2}+\rho^{2}\right)}{2 \sigma^{2}(\rho)}\right) I_{0}\left(\frac{\rho^{\prime} \rho}{\sigma^{2}(\rho)}\right) \rho^{\prime} d \rho^{\prime}\right) .
\end{aligned}
$$

We do not consider an orientation order and the loss of it here, but an averaged $\bar{g}(\rho)=\frac{1}{2 \pi} \int_{0}^{2 \pi} g(\boldsymbol{\rho}) d \varphi$ over orientation of the vector $\boldsymbol{\rho}$ (polar coordinates are used) is considered.

Thus, all the directions are equivalent, and the pare distribution function only depends on distance $\rho$. The radial distribution function $W(\rho)=\bar{g}(\rho) / n_{0}$ is usually considered. As a result, we come to the following formula:

$$
\begin{aligned}
W(\rho)=1+2 \pi \Delta(\rho)\left(\frac{1}{n_{0}} \sum_{i} N_{i} \exp (\right. & \left.-\lambda\left(\rho_{i}-D_{\min }\right)-\frac{\left(\rho_{i}^{2}+\rho^{2}\right)}{2 \sigma^{2}(\rho)}\right) I_{0}\left(\frac{\rho_{i} \rho}{\sigma^{2}(\rho)}\right)- \\
& \left.-\frac{\tilde{n}_{0}}{n_{0}} \int_{D_{\min }}^{\infty} \exp \left(-\lambda\left(\rho^{\prime}-D_{\min }\right)-\frac{\left(\rho^{\prime 2}+\rho^{2}\right)}{2 \sigma^{2}(\rho)}\right) I_{0}\left(\frac{\rho^{\prime} \rho}{\sigma^{2}(\rho)}\right) \rho^{\prime} d \rho^{\prime}\right)
\end{aligned}
$$

where summation is performed not over the nodes, but over coordination circles on which the nodes are situated, and $N_{i}$ is the number of nodes laying on each $i$-the coordination circle.

The result of the calculation is shown in Figure 3. The following parameters were used: $\sigma(\rho)=0.0877(a \rho)^{1 / 2}, n_{0}=0.965 \tilde{n}_{0}$, and $D_{\min }=0.4 a, \lambda=0.2 / a$. One can see that there is a good agreement with the experimental radial distribution function obtained by counting the number of pores in the sample shown in Figure 1a.

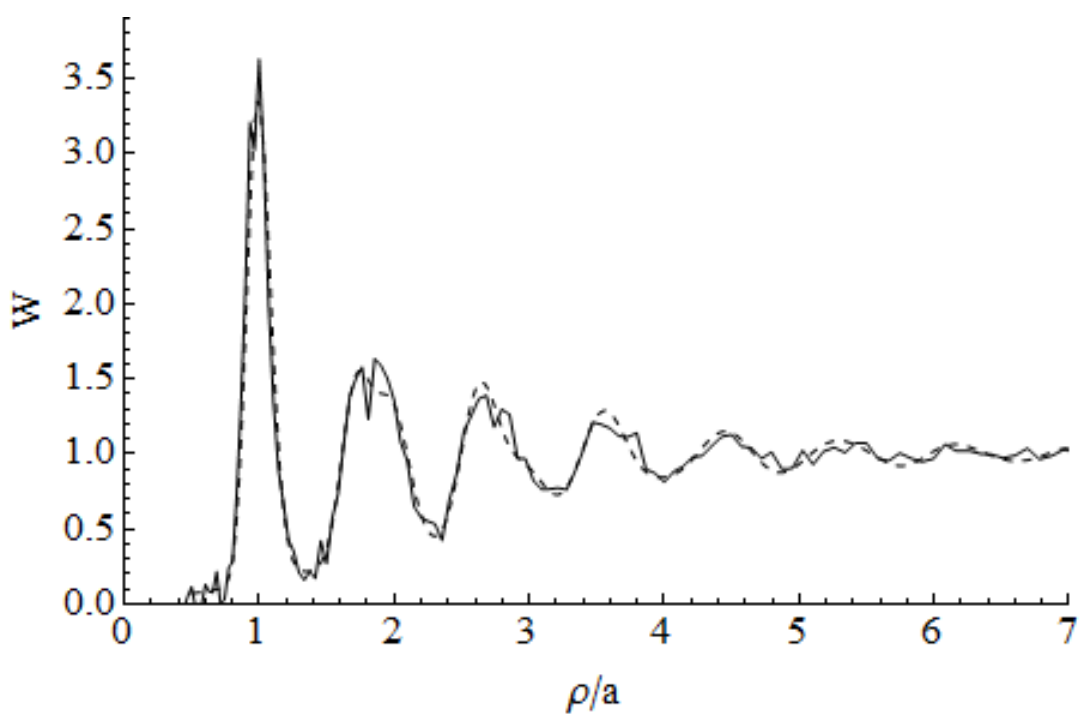

Figure 3. Radial distribution function of pores in porous aluminum oxide: experimental sample [8] (solid curve) and our calculation (dashed curve).

\section{Hard Disks in a Plane}

Let us consider a quite different system, namely, hard disk fluid. This system, on the one hand, attracts mathematicians and physicists with its simplicity, while, on the other hand, it is a test bed to explore the transition from order to disorder, hard disc liquid to hexatic, liquid to hexagonal crystal, and the liquid-to-maximally random jammed state [28-32]. The pair distribution function characterizes the important features of the system as compressibility and the equation of state [33,34]. The limiting case of a distribution function of hard disks under an increasing disk concentration is the hexagonal lattice, i.e., a two-dimensional crystal. It is reasonable that at densities 
close to the maximum, the two-particle distribution function can be described in a frame of the quasicrystal model by the smearing of a hexagonal lattice. At lower concentrations of particles, the methods of integral equations [35], based on a decoupling of the Bogolubov chain of the equations, work well. In particular, the Percus-Yevick integral equation $[29,35]$ is usually used. There question arises whether the quasicrystal model works not only in proximity to a crystal, but also at medium concentrations? Below, we consider the quasicrystal model in the range of a medium concentration of disks.

\section{Comparison with the Solutions of the Percus-Yevick Equation}

Disk concentration is characterized by the packing fraction $\eta=n_{0} \pi R^{2}$, where $R$ is a disk radius. It is evident that the value of $D_{\min }=2 R$, because the centers of the two hard disks are separated by at least this distance. The value of the lattice constant $a$ has to depend on $\eta$. The question of which lattice should be smeared to obtain the correct pair distribution functionarises. It turns out to be that the qualitative agreement with the solutions of the Percus-Yevick equation is reached if a square lattice is smeared. On the other hand, it is obvious that, under an increasing of the disk density, we should come to a hexagonal lattice. To take this into account, we suggest the following model:

$$
W(\rho)=W_{s q u}(\rho)\left(1-e^{-b \rho^{2}}\right)+W_{g e x}(\rho) e^{-b \rho^{2}},
$$

i.e., the radial distribution function is a superposition of the two terms obtained by the spreading of the square and hexagonal lattices with the weight functions depending on distance. The functions $W_{s q u}(\rho)$ and $W_{g e x}(\rho)$ are calculated by Equation (7). Parameter $\sigma(\rho)$, determining the spreading of the lattice, depends on distance as $\sigma(\rho)=\gamma \sqrt{\rho}$; that is, the nodes situated further are smeared stronger. Results of the comparison with the solutions of the Percus-Yevick equation are shown in Figures 4 and 5. It should be noted that the Percus-Yevick equation is not solved analytically for hard disks on planes, thus numerical methods were used. It seems very interesting that the "tails" of the distribution function shown in Figures $4 \mathrm{~b}$ and $5 \mathrm{~b}$ are modeled with a high accuracy. The Percus-Yevick equation stops working at a filling parameter of about 0.6 , which restricts the present consideration. However, it is evident that the parameterization of the radial distribution function given by Equation (7) will be useful for higher disk concentrations, where phase transitions occur. Table 1 contains all the parameters used in the calculations. For simplicity, we consider that the parameters $\gamma$ and $\lambda$, determining smearing and the amount of defects-vacancies, are the same for both lattices; however, constants of the lattices are taken as different. As one can see, spreading and the number of defects decrease with the increasing of $\eta$, i.e., the system tends from disorder to order.

Table 1. Constants of lattices $a_{s q u}$ and $a_{g e x}$ for modeling quasicrystal as an allocation radial distribution function at various block coefficients $\eta$, and other parameters included in Formulas (6) and (7).

\begin{tabular}{cccccc}
\hline$\eta$ & $a_{\text {squ }} /(2 R)$ & $a_{\text {gex }} /(2 R)$ & $\gamma /(2 R)^{1 / 2}$ & $2 \lambda R$ & $b(2 R)^{2}$ \\
\hline 0.62 & 1.04 & 1.1 & 0.158 & 0.027 & 0.4 \\
0.6 & 1.056 & 1.12 & 0.176 & 0.03 & 0.41 \\
0.55 & 1.095 & 1.14 & 0.192 & 0.1 & 0.45 \\
0.5 & 1.11 & 1.2 & 0.2 & 0.2 & 0.53 \\
0.45 & 1.112 & 1.3 & 0.209 & 0.5 & 0.7 \\
0.4 & 1.115 & 1.6 & 0.213 & 0.9 & 1.1 \\
0.35 & 1.117 & 2.1 & 0.224 & 1.4 & 0.75 \\
0.3 & 1.12 & 2.3 & 0.236 & 2 & $0.6^{1}$ \\
\hline
\end{tabular}

${ }^{1}$ All the quantities are dimensionless. 


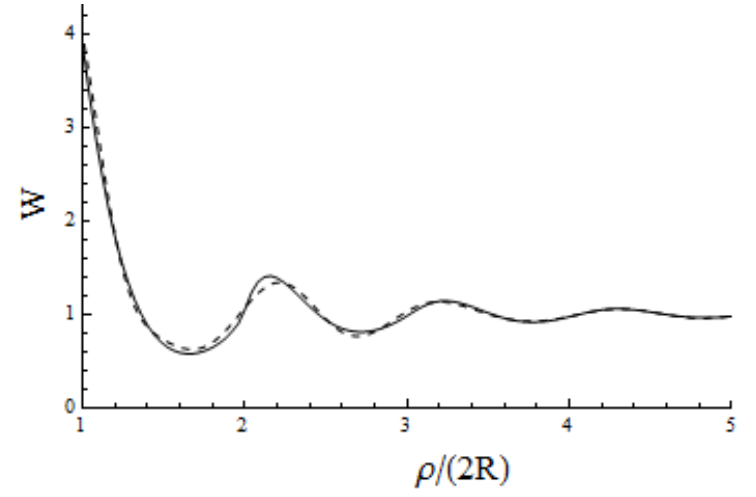

(a)

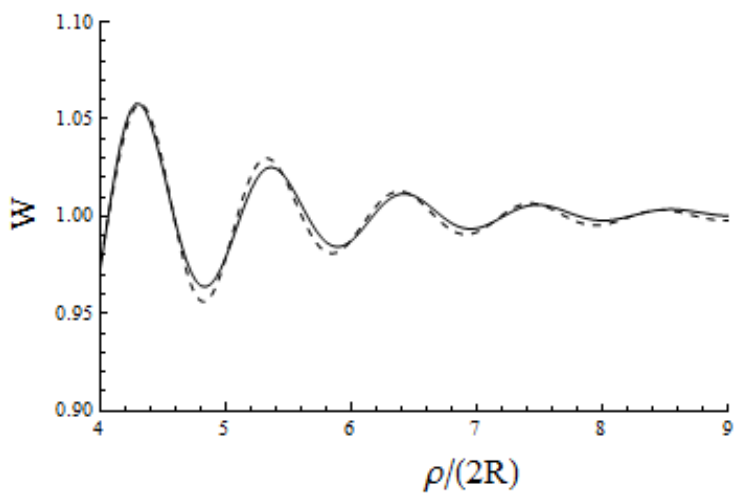

(b)

Figure 4. Radial distributions function for filling $\eta=0.62$, in a range $\rho \in\{1,5\}$ (a) and in a range $\rho \in\{4,9\}$ ("a tail of the distribution function") (b). Solid line is a solution of the Percus-Yevick equation, and dashed line is the quasicrystal model.

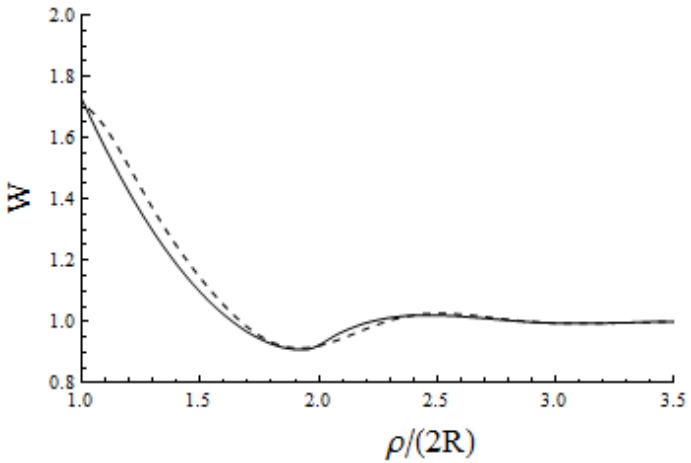

(a)

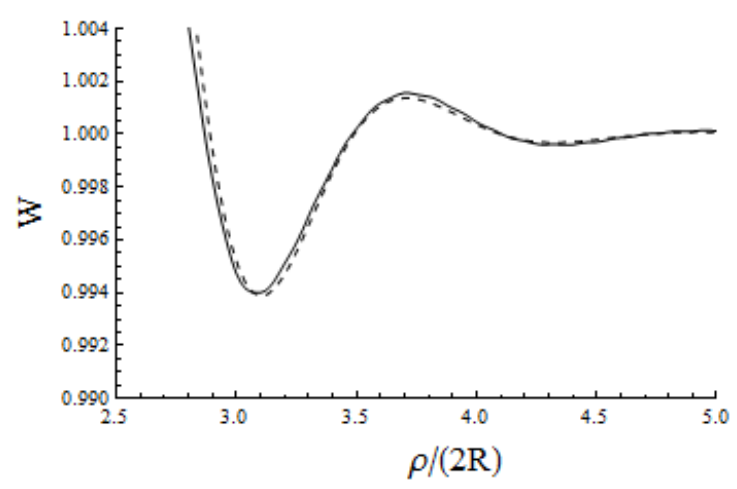

(b)

Figure 5. Radial distribution functions for filling $\eta=0.3$, in a range $\rho \in\{1,3.5\}$ (a) and in a range $\rho \in\{2.5,5.0\}$ ("a tail of the distribution function") (b). Solid line is a solution of the Percus-Yevick equation, and dashed line is the quasicrystal model.

\section{Conclusions}

Radial distribution function parameterization, based on the quasicrystal model, is presented. This parameterization describes the same footing as porous aluminum oxide, so as 2-D hard disk fluid. The quasicrystal model is based on two effects: the first one is the vacancy-type defects formation and the second one is the destroying of the crystal lattice by smearing. The successes of this parametrization show that even liquid of the hard disks contains features of a crystal. The model will serve as a tool for the description of the phase transitions in 2-D fluids. On the other hand, it could be the base for variational methods, because the parameters describing spreading, defects probability, and lattice constants could be found by the minimization of some function [36].

Conflicts of Interest: The authors declare no conflict of interest.

\section{References}

1. Peierls, R.E. Transformation tempretures. Helv. Phys. Acta Suppl. 1934, 2, 81-83.

2. Landau, L.D. On the theory of phase transitions I. Phys. Z. Sowjetunion 1937, 11, $26-35$.

3. Landau, L.D. On the theory of phase transitions II. Phys. Z. Sowjet. 1937, 11, 545-555.

4. Mermin, N.D. Crystalline order in two dimensions. Phys. Rev. 1968, 176, 250-254.

5. Eder, F.R.; Kotakoski, J.; Kaiser, U.; Meyer, J.C.A journey from order to disorder-Atom by atom transformation from graphene to 2D carbon glass. Sci. Rep. 2014, 4, 4060-4066, doi:10.1038/srep04060. 
6. Warren, B.E. X-ray Diffraction Study of the Structure of Glass. Chem. Rev. 1940, 26, 237-255, doi:10.1021/cr60084a007.

7. Wright, A.C.; Thorpe, M.F. Eighty years of random networks. Phys. Status Solidi B 2013, 250, 931-936, doi:10.1002/pssb.201248500.

8. Prins, G.A.; Petersen, H. Theoretical diffraction patterns corresponding to some simple types of molecular arrangement in liquids. Physica 1936, 3, 147-153.

9. Glauberman, A.E. On the theory of a local order inliquids. Zh. Eksp. Teor. Fiz. 1952, 22, 249-250.

10. Tsvetkov, V.P. About structure of the liquid metals. Izv. Vyss. Uchebn. Zaved. Ser. Fiz. 1960, 1, 145-154.

11. Franchetti, S. On a model for monoatomic liquids. Nuovo Cim. B 1968, 55, 335-347.

12. Medvedev, N.N.; Naberukhin, Y.I. Description of the radial distribution function of liquid argon in the quasi-crystalline model of liquids. Phys. Chem. Liq. 1978, 8, 167-187, doi:10.1080/00319107808084751.

13. Baer, S. Form of the radial distribution function and the structure factor, derived from the "structural diffusion" model for liquids. Phys. A 1978, 91, 603-611.

14. Skryshevskii, A.F. Structural Analysis of Liquids and Amorphous Solids; Vysshaya Shkola: Moscow, Rassia, $1980 ;$ p. 49.

15. Medvedev, N.N.; Naberukhin, Y.I.; Semenova, I.Y. The radial distribution function and structure factor of liquid and amorphous gallium as described by the quasi-crystalline model. J. Non-Cryst. Solids 1984, 64, 421-432, doi:10.1016/0022-3093(84)90193-90195.

16. Miskevich, A.A.; Loiko, V.A. Coherent transmission and reflection of a two-dimensional planar photonic crystal. Zh. Eksp. Teor. Fiz. 2011, 140, 5-20.

17. Cherkas, N.L.; Cherkas, S.L. Model of the radial distribution function of pores in a layer of porous aluminum oxide. Crystallogr. Rep. 2016, 61, 285-290.

18. Masuda, H.; Fukuda,K. Ordered metal nanohole arrays made by a two-step replication of honeycomb structures of anodic alumina. Science 1995,268, 1466-1468, doi:10.1126/science.268.5216.1466.

19. Jessensky, O.; Müller, F.; Gösele, U. Self-organized formation of hexagonal pore arrays in anodic alumina. Appl. Phys. Lett. 1998, 72, 1173-1175, doi:10.1063/1.121004.

20. Nielsch, K.; Choi, J.; Schwirn, K. Self-ordering Regimes of Porous Alumina: The 10 Porosity Rule. Nano Lett. 2002, 2, 677-680.

21. Parkhutik, V.P.; Shershulsky, V.I. Theoretical modelling of porous oxide growth on aluminium. J. Phys. D 1992, 25,1258-1263, doi:10.1088/0022-3727/25/8/017.

22. Singh, G.K.; Golovin, A.A.; Aranson, I.S. Formation of self-organized nanoscale porous structures in anodic aluminum oxide. Phys. Rev. B 2006, 73, 205422, doi:10.1103/PhysRevB.73.205422.

23. Cherkas, N.L. Electromagnetic wave in a medium consisting of parallel dielectric cylinders. Opt. Spektrosk. 1996, 81, 990-996.

24. Yakovleva, N.M.; Yakovlev, A.N.; Gafiyatullin, M.M. Computer diagnostics of the mesoscopic structure of the nanoporous oxides of aluminum. Zavod. Lab. Diagn. Mater. 2009, 75, 21-26.

25. Kaatz, F.H. Measuring the order in ordered porous arrays: Can bees outperform humans? Naturwissenschaften 2006, 93, 374-378.

26. Vodopivec, A.; Kaatz, F.H.; Mohar, B. Topographical distance matrices for porous arrays. J. Math. Chem. 2010, 47, 1145-1153.

27. Lutich, A.A.; Gaponenko, S.V.; Gaponenko, N.V.; Molchan, I.S.; Sokol, V.A.; Parkhutik, V. Anisotropic light scattering in nanoporous materials: A photon density of states effect. Nano Lett. 2004, 4, 1755-1758.

28. Fernandez, J.F.; Alonso, J.J.; Stankiewicz, J. Melting of systems of hard disks by Monte Carlo simulations. Phys. Rev. E 1997, 55, 750-764, doi:10.1103/physreve.55.750.

29. Adda-Bedia, M.; Katzav, E.; Vella, D. Solution of the Percus-Yevick equation for hard disks. J. Chem. Phys. 2008, 128, 184508, doi:10.1063/1.2919123.

30. Kapfer, S.C.; Krauth, W. Sampling from a polytope and hard-disk Monte Carlo. J. Phys. Conf. Ser. 2013, 454, 012031, doi:10.1088/1742-6596/454/1/012031.

31. Gaal, A.T. Long-range order in a hard disk model. Electron. Comm. in Probab. 2014, 19, 1-9, doi:10.1214/ecp.v19-3047.

32. Atkinson, S.; Stillinger, F.H.; Torquato, S. Existence of isostatic, maximally random jammed monodisperse hard-disk packings. Proc. Natl. Acad. Sci. USA 2014, 111, 18436-18441, doi:10.1073/pnas.1408371112. 
33. Engel, M.; Anderson, J.A.; Glotzer, S.C.; Isobe, M.; Bernard, E.P.; Krauth, W. Hard-disk equation of state: First-order liquid-hexatic transition in two dimensions with threesimulation methods. Phys. Rev. E 2013, 87, 042134, doi:10.1103/physreve.87.042134.

34. Sokołowski,S. A note on the two-dimensional radial distribution function. Czechoslov. J. Phys. B 1978, 28, 713-720, doi:10.1007/bf01595993.

35. Kovalenko, N.P.; Fisher, I.Z. Method of integral equations in statistical theory of liquids. UspekhiFizicheskihNauk 1972, 108, 209-239, doi:10.3367/ufnr.0108.197210a.0209.

36. Arinshtein, E.A. A model of the liquid-crystal phase transition and the quasicrystal model of liquid. Theor. Math. Phys. 2007, 151, 571-585, doi:10.1007/s11232-007-0043-y.

(C) 2018 by the authors. Licensee MDPI, Basel, Switzerland. This article is an open access article distributed under the terms and conditions of the Creative Commons Attribution (CC BY) license (http://creativecommons.org/licenses/by/4.0/). 